\title{
A prevalência de incontinência urinária em mulheres praticantes de musculação: uma
}

\section{revisão de literatura}

\author{
The prevalence of urinary incontinence in women practicing bodybuilding: a literature review \\ La prevalencia de la incontinencia urinaria en mujeres que practican el bodybuilding: una revisión de
}

\section{la literatura}

Recebido: 22/09/2021 | Revisado: 26/09/2021 | Aceito: 27/09/2021 | Publicado: 28/09/2021

\author{
Carla Bianca Santos da Silva \\ ORCID: https://orcid.org/0000-0003-4654-9325 \\ Centro Universitário da Amazônia, Brasil \\ E-mail: c.biancasantos23@gmail.com \\ Rafaela da Silva Nascimento \\ ORCID: https://orcid.org/0000-0002-0339-4899 \\ Centro Universitário da Amazônia, Brasil \\ E-mail: rafasilva1830@gmail.com \\ Indiara de Alencar \\ ORCID: https://orcid.org/0000-0003-3204-3278 \\ Universidade Estado do Pará, Brasil \\ E-mail: indiara.alencarstm@gmail.com
}

\begin{abstract}
Resumo
A musculação está se tornando um dos fatores de riscos consideráveis para a existência de Incontinência Urinária (IU) em mulheres que praticam a atividade física. Isso se deve aos maiores esforços, como é o caso dessa modalidade, podendo gerar um aumento da pressão intra-abdominal, sobrecarregando os órgãos da pelve e, consequentemente, provocando danos na musculatura responsável por esses órgãos, causando assim a IU, além do mais, o excesso desse exercício pode provocar fadiga dos músculos do assoalho pélvico, predispondo assim a IU. Com isso, o estudo buscou informações em referências bibliográficas de estudo práticos e estudo que foram realizados através de questionários online a respeito da prevalência de perda de urina em mulheres que desenvolvem a musculação, tendo assim um número amostral considerado para realizar uma conclusão acerca do problema proposto. De acordo com estudo podemos observar que em sua grande maioria a perda de urina prevaleceu em alguns casos e que gera desconforto e influência diretamente na qualidade vida das mulheres. Dessa maneira, o estudo constatou que, apesar das poucas mulheres estudadas, sendo 918 praticantes da atividade, destas 152 apresentaram perda de urina, sendo assim, há à necessidade de enfatizar que os profissionais que atuam nessa área devem elaborar melhores estratégias e formas mais adequadas no planejamento de treino das alunas praticantes da musculação.

Palavras-chave: Incontinência urinária; Musculação; Prevalência; Mulheres.
\end{abstract}

\begin{abstract}
Weight training is becoming one of the considerable risk factors for the existence of Urinary Incontinence (UI) in women who practice physical activity. This is due to greater efforts, as is the case of this modality, which can generate an increase in intra-abdominal pressure, overloading the pelvic organs and, consequently, causing damage to the muscles responsible for these organs, thus causing UI, in addition, the excess of this exercise can cause fatigue of the pelvic floor muscles, thus predisposing to UI. Thus, the study sought information in bibliographic references of practical studies and studies that were carried out through online questionnaires about the prevalence of urine loss in women who develop bodybuilding, thus having a sample number considered to reach a conclusion about the problem proposed. According to a study, we can observe that the vast majority of urine loss prevailed in some cases and that it generates discomfort and directly influences the quality of life of women. Thus, the study found that, despite the few women studied, being 918 practitioners of the activity, of these 152 had urine loss, so there is a need to emphasize that professionals working in this area should develop better strategies and more appropriate ways in the training planning of the students who practice bodybuilding.
\end{abstract}

Keywords: Urinary incontinence; Bodybuilding; Prevalence; Women.

\section{Resumen}

El entrenamiento con pesas se está convirtiendo en uno de los factores de riesgo considerables para la existencia de incontinencia urinaria (IU) en mujeres que practican actividad física. Esto se debe a mayores esfuerzos, como es el caso de esta modalidad, que puede generar un aumento de la presión intraabdominal, sobrecargando los órganos pélvicos y, en consecuencia, provocando daño a los músculos responsables de estos órganos, provocando así IU, además, el exceso de este ejercicio puede provocar fatiga de la musculatura del suelo pélvico, predisponiendo así a la IU. Así, el estudio buscó información en referencias bibliográficas de estudios prácticos y estudios que se realizaron a través de cuestionarios online 
sobre la prevalencia de pérdida de orina en mujeres que desarrollan culturismo, teniendo así un número de muestra considerado para llegar a una conclusión sobre el problema propuesto. Según un estudio, podemos observar que la gran mayoría de la pérdida de orina prevaleció en algunos casos y que genera malestar e influye directamente en la calidad de vida de las mujeres. Así, el estudio encontró que, a pesar de las pocas mujeres estudiadas, siendo 918 practicantes de la actividad, de estas 152 tuvieron pérdida de orina, por lo que es necesario enfatizar que los profesionales que trabajan en esta área deben desarrollar mejores estrategias y formas más adecuadas en la planificación de la formación de los estudiantes que practican el culturismo.

Palabras clave: Incontinencia urinaria; Culturismo; Predominio; Mujeres.

\section{Introdução}

Segundo a International Continence Society (ICS) estabelece que a Incontinência Urinária (IU) é qualquer perda espontânea de urina. Sendo categorizada em três tipos, Incontinência Urinária por Urgência (IUU) onde as perdas de urina estão relacionadas a episódios de uma vontade súbita e intensa de urinar, a Incontinência Urinária Esforço (IUE) que é estabelecida como perda involuntária de urina aos esforços, tosse ou espirro e afeta de forma negativa a vida das mulheres sendo a ocorrência mais comum entre as mulheres, e a Incontinência Urinaria Mista (IUM) que está ligada a combinação de sintomas da incontinência urinária de esforço e de incontinência urinária de urgência (Naves et al., 2016).

O Brasil tem hoje uma população de cerca de 211 milhões de habitantes, dos quais aproximadamente 56\% são mulheres, e por volta de um terço da população feminina é atingida pela IU. Essa afecção contém índices significativos de prevalência mundial também, provocando em ambos os gêneros, com prevalência de 27,6\% no gênero feminino e 10,5\% no masculino, sendo mais frequente nas mulheres por conta da própria anatomia e do fator idade (Mourão et al., 2017).

A IU corresponde a uma patologia no qual resulta em diversos efeitos sobre as atividades diárias como interação social e percepção da própria saúde. Podemos relacionar a IU com o bem-estar social e mental, com isso, abrangendo problemas na vida sexual, social, e outros, como baixa autoestima e depressão (Lopes et al., 2006).

Mesmo com o conhecimento acerca da seriedade da IU, a grande maioria das mulheres incontinentes manifestam repercussões psicológicas como: incapacidades, perda da autoestima, vergonha dos episódios de perda de urina em ambientes públicos, receio que as pessoas sintam o odor da urina e de perder o emprego. Por esses motivos, há uma mudança hábitos diários para remediar a incontinência, o que dificulta o diagnóstico da doença, pois grande parte das mulheres sente vergonha de relatar esse fato aos profissionais da saúde, familiares e amigos (Higa et al., 2010).

Acrescentado a isso, a população de idosas acometidas pela IU sofre interferência de forma considerável na qualidade de vida, pois causa constrangimento social, que possivelmente tira a segurança da idosa em um ambiente social devido o desconforto causado, resultando em um sentimento de vergonha, isolamento, estresse, baixa autoestima, além de interferir também em sua vida doméstica devido às limitações que o indivíduo irá apresentar em suas atividades de vida diária (Silva et al., 2019).

Sendo assim é importante a verificação das causas da IU, tendo assim, as causas mais comumente encontradas, as quais podem ser agrupadas em dois grupos: as resultantes de alterações vesicais primárias ou secundárias; e as decorrentes de distúrbios uretrais. As alterações vesicais são especialmente representadas pela hiperatividade da musculatura detrusora, identificada por contrações involuntárias desse músculo, que acabam ocasionando na eliminação involuntária da urina armazenada. A hiperatividade do detrusor pode ser ramificada em dois tipos: fásica e terminal. Na hiperatividade fásica, a extensão das contrações aumenta de acordo com o volume vesical e podem, ou não, ocasionar à IU. Por outro lado, na hiperatividade terminal, há uma única e involuntária contração do detrusor, que não pode ser suprimida e invariavelmente resulta em IU, com esvaziamento por completo da bexiga (Cândido et al., 2017)

Nesse sentido, é importante a verificação da existência de algumas evidências de que a incontinência urinaria é associada a esportes de alto impacto, como por exemplo, o voleibol, basquetebol, corrida de longa distância, saltos, musculação e crossfit acaba tendo aumento na probabilidade do aparecimento de perdas significativas de urina. Além disso, os praticantes tendem a 
afastar-se de suas rotinas esportivas, por conta de constrangimentos que a incontinência atribui com pontos negativos tanto a sua saúde física quanto psicossocial, gerando limitações no âmbito emocional, social e sexual e afetando frequentemente na qualidade de vida (Prigol et al., 2014).

Apesar dos benefícios que a atividade física proporciona à saúde do ser humano, é necessário a verificação da existência de causa da IU em alguns esportes. Contudo, é válido ressaltar que algumas atividades físicas de moderada intensidade já foram demonstradas como efetivas na diminuição das chances de desenvolver IU, entretanto, o exercício físico intenso é listado como um dos principais fatores de risco para o surgimento do problema de forma que há indícios de que esportes de alto impacto podem resultar em alteração das pressões intra-abdominais refletidas aos órgãos pélvicos, induzindo a IUE durante o treino esportivo ou competições (Ferraz et al., 2018).

Por conta dessa prática, ainda faltam informações sobre a importância da contração da musculatura perineal no exercício físico, já que a prevalência de IU pode chegar a $80 \%$ em atletas de elite, enquanto aqueles exercícios em mulheres com idade média de 15 a 64 anos. Para idosos com atividades simples, pode variar de 10\% a 55\% (Borghetti et al., 2007).

Porém, embora estudos tenham apontado o risco de IU entre praticantes de exercícios físicos, principalmente atletas, ainda não está claro se mulheres que se exercitam em academia apresentam risco de IU. A incontinência urinária é um problema que afeta muitas pessoas em todo o mundo (Da silva et al., 2018).

Contudo, a IU tem tratamento que pode ser conservador ou cirúrgico. No entanto, o tratamento cirúrgico tem o custo financeiro mais elevado e, por ser mais invasivo, poderá ocorrer complicações, além de ser contraindicado em alguns casos e, por muitas vezes, ser negado pela própria paciente (Guerra et al., 2014).

Quanto ao tratamento conservador fisioterapêutico para a incontinência urinaria, no qual é conduzido para a atividade dos músculos pélvicos nas incontinências de esforço, de urgência e mistas. A fisioterapia se revela um recurso terapêutico eficaz, não sendo desagradável ou gerando risco, compatível com outros recursos de tratamento. Envolve uma tarefa específica de treino de percepção corporal e de normalização do tônus dos músculos pélvicos (Henkes et al., 2015).

Com base no exposto acima, apesar do pequeno número de mulheres incontinentes pela prática da musculação é necessário realizar uma revisão literária para identificar a prevalência de incontinência urinária em mulheres praticantes de musculação, visto que há o aumento da prática de exercícios pela população feminina e pela importância da exploração deste tema.

\section{Metodologia}

A construção do presente estudo fundamentou-se em uma revisão bibliográfica de pesquisa com abordagem quantitativa, de natureza básica com o caráter descritivo.

A coleta de dados ocorreu através do levantamento das produções científicas sobre a prevalência de IU em mulheres praticantes de musculação, produzidas entre os anos de 2014 a 2021. Para ter resultados dessa revisão tem como questão problema sobre qual "a prevalência de incontinência urinaria em mulheres praticantes de musculação".

A revisão foi realizada a partir de artigos científicos relacionados ao tema proposto, realizada assim selecionados artigos, avaliações de estudos para construção, resultados e as discussões. Os artigos foram pesquisados na base eletrônica Google Acadêmico, Scientific Electronic Library Online (SciElo), Repositório Acadêmico Ânima (RUNA), utilizando palavras chaves como: incontinência urinaria, prevalência, mulheres, praticantes de musculação.

A análise foi realizada com leituras criteriosas dos artigos selecionados, sendo um total de 10 estudos, onde foi verificado e dado ênfase sobre o tema proposto: A prevalência da incontinência em mulheres praticantes de musculação.

Os artigos foram selecionados segundo os seguintes critérios de inclusão: terem sido publicados no período de 2014 a 2021 , estarem em linguagem inglesa e portuguesa, abordar temas relacionados à IU em mulheres que praticam musculação, publicações cientificas e de livre acesso. 
Excluíram-se os artigos que enfatizavam IU masculina, infantil e grávidas, incontinência fecal, trabalhos não concluídos e artigos que não é compatível com o tema.

\section{Resultados e Discussão}

No Quadro 1 mostra-se 10 estudos realizado só em mulheres, sendo no total 918 mulheres que praticam atividade física e que foram submetidas aos estudos para a realização de verificação de IU presente ou não no ato da musculação.

Quadro 1 - Resultados dos estudos entre os anos de 2014 à 2021.

\begin{tabular}{|c|c|c|c|c|c|}
\hline Título & Autor/Ano & Amostra & Idade & Objetivo & Resultados/Conclusão \\
\hline $\begin{array}{l}\text { Impacto dos sinais e } \\
\text { sintomas de } \\
\text { incontinência } \\
\text { urinária na } \\
\text { qualidade de vida } \\
\text { de mulheres } \\
\text { praticantes de } \\
\text { musculação no } \\
\text { Estado de Sergipe. }\end{array}$ & $\begin{array}{l}\text { SOUSA, } \\
\text { D.S,et al, } \\
2021 .\end{array}$ & $\begin{array}{c}52 \\
\text { mulheres }\end{array}$ & $\begin{array}{l}\text { Em média } \\
27,11 \text { anos }\end{array}$ & $\begin{array}{l}\text { Analisar a } \\
\text { prevalência de sinais } \\
\text { e sintomas de } \\
\text { Incontinência } \\
\text { Urinária por esforço } \\
\text { em mulheres } \\
\text { praticantes de } \\
\text { musculação. }\end{array}$ & $\begin{array}{l}\text { Os sinais e sintomas da incontinência urinária } \\
\text { estavam presentes nas mulheres praticantes de } \\
\text { musculação, evidenciando o impacto de grave a } \\
\text { muito grave ocasionado na qualidade de vida } \\
\text { destas. }\end{array}$ \\
\hline $\begin{array}{l}\text { Sintomas de perda } \\
\text { urinária em } \\
\text { mulheres } \\
\text { praticantes de } \\
\text { exercícios físicos de } \\
\text { alto e baixo } \\
\text { impacto. }\end{array}$ & $\begin{array}{c}\text { ALVES, F D. } \\
\text { A, } 2021 .\end{array}$ & $\begin{array}{c}48 \\
\text { mulheres }\end{array}$ & $\begin{array}{c}\text { Entre } 37 \text { a } \\
45 \text { anos }\end{array}$ & $\begin{array}{l}\text { Identificar e } \\
\text { evidenciar a } \\
\text { presença de perda } \\
\text { urinaria em mulheres } \\
\text { praticantes de } \\
\text { exercício físico para } \\
\text { a associação da } \\
\text { presença dos } \\
\text { sintomas urinários } \\
\text { com o tipo exercício } \\
\text { físico realizado. }\end{array}$ & $\begin{array}{l}\text { Embora não tenha havido diferença estatística } \\
\text { em relação a ocorrência perda urinaria, o grupo } \\
\text { de praticantes de corrida de rua apresentou } \\
\text { maior índice de perda urinaria, onde pode estar } \\
\text { relacionado a fadiga muscular devido a } \\
\text { frequência e tempo de pratica do exercício que } \\
\text { se demonstrou maior em relação ao grupo } \\
\text { musculação, pois é considerada uma modalidade } \\
\text { de alto impacto que ocasiona abruptos aumentos } \\
\text { de pressões constantes. }\end{array}$ \\
\hline $\begin{array}{l}\text { Diferença na } \\
\text { prevalência de } \\
\text { incontinência } \\
\text { urinária entre } \\
\text { mulheres } \\
\text { praticantes de } \\
\text { musculação ou } \\
\text { crossfit. }\end{array}$ & $\begin{array}{l}\text { BATTISTEL, } \\
\text { G. F, } 2020 .\end{array}$ & $\begin{array}{c}220 \\
\text { mulheres }\end{array}$ & $\begin{array}{l}\text { Igual ou } \\
\text { superior a } \\
18 \text { anos }\end{array}$ & $\begin{array}{l}\text { Analisar a diferença } \\
\text { na prevalência do } \\
\text { autorrelato de } \\
\text { incontinência } \\
\text { urinária (IU) em } \\
\text { mulheres que } \\
\text { praticam musculação } \\
\text { e as de praticantes } \\
\text { crossfit. }\end{array}$ & $\begin{array}{l}\text { Este estudo, não encontrou diferença na } \\
\text { prevalência de IU ao comparar mulheres } \\
\text { praticantes de crossfit e musculação. Também } \\
\text { não apresentou associação significativa entre a } \\
\text { perda urinária e os fatores de risco sabidamente } \\
\text { reconhecidos na literatura. Entretanto viu-se que } \\
\text { IU tende a ser mais severa nas praticantes de } \\
\text { atividades físicas de alto impacto. }\end{array}$ \\
\hline $\begin{array}{c}\text { Prevalência de } \\
\text { Incontinência } \\
\text { Urinária em } \\
\text { Mulheres } \\
\text { Praticantes de } \\
\text { Pilates e de } \\
\text { Musculação. }\end{array}$ & $\begin{array}{l}\text { AMORIM, L. } \\
\text { F. D. et al, } \\
2019\end{array}$ & $\begin{array}{c}60 \\
\text { mulheres }\end{array}$ & $\begin{array}{c}20 \text { a } 50 \\
\text { anos }\end{array}$ & $\begin{array}{c}\text { Identificar a } \\
\text { prevalência de } \\
\text { incontinência } \\
\text { Urinária em } \\
\text { mulheres praticantes } \\
\text { de pílates e de } \\
\text { Musculação. }\end{array}$ & $\begin{array}{l}\text { Foram encontrados um índice de IU maior nas } \\
\text { praticantes de musculação, porém, as praticantes } \\
\text { de pilates apresentaram IU de maior impacto. } \\
\text { Desta forma, este trabalho indica que se tenha } \\
\text { mais atenção e cuidado, seja pelos praticantes ou } \\
\text { pelos profissionais orientadores de musculação, } \\
\text { quando comparada com a amostra de pilates, se } \\
\text { mostrou mais suscetível a IU. }\end{array}$ \\
\hline $\begin{array}{c}\text { Disfunções } \\
\text { urinárias em } \\
\text { mulheres } \\
\text { praticantes de } \\
\text { atividade física em } \\
\text { academias - um } \\
\text { estudo transversal }\end{array}$ & $\begin{array}{l}\text { SILVA, L. B. } \\
\text { D. et al, } 2018\end{array}$ & $\begin{array}{c}147 \\
\text { mulheres }\end{array}$ & $\begin{array}{c}25 \text { a } 55 \\
\text { anos }\end{array}$ & $\begin{array}{c}\text { Verificar a } \\
\text { prevalência de } \\
\text { IUE em mulheres } \\
\text { com prática regular } \\
\text { de atividade física } \\
\text { em academias }\end{array}$ & $\begin{array}{l}\text { A prevalência de IU em mulheres praticantes de } \\
\text { exercícios aeróbicos e musculação nas } \\
\text { academias foi baixa ( } 12 \%) \text {, com severidade de } \\
\text { moderada a grave e sem associação com idade, } \\
\text { modalidade, tempo de prática, turno e frequência } \\
\text { de exercícios. }\end{array}$ \\
\hline $\begin{array}{l}\text { Incontinência } \\
\text { Urinária em } \\
\text { Mulheres } \\
\text { Praticantes de } \\
\text { Musculação } \\
\end{array}$ & $\begin{array}{l}\text { MELO, A.T. } \\
\text { D; } \\
\text { CIRQUEIRA, } \\
\text { R. P, } 2018 .\end{array}$ & $\begin{array}{c}30 \\
\text { mulheres }\end{array}$ & $\begin{array}{c}\text { A partir de } \\
18 \text { anos }\end{array}$ & $\begin{array}{l}\text { Verificar a presença } \\
\text { de incontinência } \\
\text { urinaria em mulheres } \\
\text { praticantes de } \\
\text { musculação. }\end{array}$ & $\begin{array}{c}\text { Não houve associação estatisticamente } \\
\text { significante entre a prática de musculação e a } \\
\text { incontinência urinaria. }\end{array}$ \\
\hline $\begin{array}{l}\text { Prevalência de } \\
\text { incontinência } \\
\text { urinária de esforço e }\end{array}$ & $\begin{array}{l}\text { NUNES, I; } \\
\text { BRUNAZO, } \\
\text { P.F, } 2017 . \\
\end{array}$ & $\begin{array}{c}39 \\
\text { mulheres }\end{array}$ & $\begin{array}{c}18 \text { a } 50 \\
\text { anos }\end{array}$ & $\begin{array}{c}\text { Analisar a } \\
\text { prevalência de } \\
\text { incontinência }\end{array}$ & $\begin{array}{l}\text { As praticantes de jump tiveram uma perda } \\
\text { urinária objetiva maior quando comparadas as } \\
\text { praticantes de musculação, contudo as mulheres }\end{array}$ \\
\hline
\end{tabular}




\begin{tabular}{|c|c|c|c|c|c|}
\hline $\begin{array}{l}\text { função dos } \\
\text { músculos do } \\
\text { assoalho pélvico em } \\
\text { mulheres } \\
\text { praticantes de jump } \\
\text { ou musculação }\end{array}$ & & & & $\begin{array}{l}\text { urinária de esforço } \\
\text { (IUE) e a função dos } \\
\text { músculos do } \\
\text { assoalho pélvico } \\
\text { (MAP) em mulheres } \\
\text { praticantes de jump } \\
\text { ou musculação. }\end{array}$ & $\begin{array}{l}\text { praticantes de ambas modalidades apresentaram } \\
\text { uma reduzida função dos músculos do assoalho } \\
\text { pélvico. Além disso nota-se que em ambas as } \\
\text { modalidades as mulheres que perdem urina } \\
\text { durante o exercício perdem também no dia a dia. } \\
\text { Isso pode estar relacionado com a reduzida } \\
\text { função dos músculos do assoalho pélvico que as } \\
\text { mesmas apresentaram. }\end{array}$ \\
\hline $\begin{array}{l}\text { Avaliação da } \\
\text { qualidade de vida } \\
\text { em mulheres com } \\
\text { sintomas de } \\
\text { incontinência } \\
\text { urinária de esforço } \\
\text { praticantes de } \\
\text { atividade física. }\end{array}$ & $\begin{array}{l}\text { NAVES, P. P. } \\
\text { et al, } 2016 .\end{array}$ & $\begin{array}{c}42 \\
\text { mulheres }\end{array}$ & $\begin{array}{l}\text { Em média } \\
32,7 \text { anos }\end{array}$ & $\begin{array}{l}\text { Identificar os } \\
\text { sintomas da } \\
\text { incontinência } \\
\text { urinária de esforço } \\
\text { em mulheres } \\
\text { praticantes de } \\
\text { atividade física, e a } \\
\text { qualidade de vida } \\
\text { das mesmas. }\end{array}$ & $\begin{array}{c}\text { O presente estudo sugere que mulheres } \\
\text { praticantes de atividade física, podem apresentar } \\
\text { queixas de sintomas urinários. As atividades de } \\
\text { impacto é um fator de risco importante para o } \\
\text { desenvolvimento da IUE, o que pode influenciar } \\
\text { de forma negativa a qualidade de vida destas } \\
\text { mulheres. }\end{array}$ \\
\hline $\begin{array}{l}\text { Prevalência da } \\
\text { incontinência } \\
\text { urinária em } \\
\text { mulheres } \\
\text { praticantes de } \\
\text { atividade física nas } \\
\text { academias da cidade } \\
\text { de Erechim }\end{array}$ & $\begin{array}{c}\text { PRIGOL, S. et } \\
\text { al, } \\
2014\end{array}$ & $\begin{array}{c}172 \\
\text { mulheres }\end{array}$ & $\begin{array}{c}20 \text { a } 40 \\
\text { anos }\end{array}$ & $\begin{array}{c}\text { Verificar a } \\
\text { prevalência da IU em } \\
\text { mulheres durante a } \\
\text { prática de atividade } \\
\text { física nas academias } \\
\text { da cidade de } \\
\text { Erechim, identificar } \\
\text { qual a atividade } \\
\text { física que mais gera } \\
\text { IU nas mulheres e } \\
\text { verificar se a IU } \\
\text { interfere na } \\
\text { qualidade de vida } \\
\text { (QV) das mulheres } \\
\text { praticantes de } \\
\text { atividade física. }\end{array}$ & $\begin{array}{l}\text { Verificou-se que houve uma baixa prevalência } \\
\text { da IU nas mulheres praticantes de atividade } \\
\text { física. Dentre as atividades pesquisadas, a } \\
\text { realização na cama elástica (jump) mostrou-se } \\
\text { ser a que mais ocasionou a perda de urina nas } \\
\text { mulheres jovens. }\end{array}$ \\
\hline $\begin{array}{l}\text { Incontinência } \\
\text { urinária em } \\
\text { mulheres jovens } \\
\text { praticantes de } \\
\text { exercício físico }\end{array}$ & $\begin{array}{l}\text { PATRIZZI, L } \\
\text { J. et al, } 2014 .\end{array}$ & $\begin{array}{c}108 \\
\text { mulheres }\end{array}$ & $\begin{array}{c}18 \text { a } 30 \\
\text { anos }\end{array}$ & $\begin{array}{l}\text { Comparar a } \\
\text { prevalência do } \\
\text { autorrelato de } \\
\text { incontinência } \\
\text { urinária em mulheres } \\
\text { jovens de acordo } \\
\text { com as modalidades } \\
\text { de exercício físico. }\end{array}$ & $\begin{array}{c}\text { A IU está presente nas mulheres em grande } \\
\text { percentual nas diferentes modalidades } \\
\text { esportivas, com associação significativa na } \\
\text { musculação }(\mathrm{p}=0,01) \text {. Desta forma, é necessário } \\
\text { enfatizar que os profissionais que atuam nessa } \\
\text { área devem elaborar melhores estratégias de } \\
\text { orientação às mulheres durante a execução do } \\
\text { exercício físico, esclarecendo o risco de } \\
\text { desenvolver IU. }\end{array}$ \\
\hline
\end{tabular}

Fonte: Silva, Nascimento e Alencar (2021).

No presente estudo foi analisado a prevalência de incontinência urinária em mulheres praticantes de musculação, apesar de alguns estudos citarem a modalidade Jump como grande causador da IU, a musculação tem sido frequente causadora de IU variando de baixa, média e alta prevalência de IU nas mulheres que foram submetidas ao estudo, impactando, consequentemente em sua qualidade de vida. As causas e sintomas de perda urinária em mulheres praticantes de musculação vem se tornando um objeto de estudo frequente, uma vez que pode ser constatado o crescimento dessa população em relação à prática, seja por motivos estéticos ou de saúde.

No estudo de Sousa et al., 2021 demonstrou que 6 mulheres de 52 amostras constataram ter a incontinência urinária e que são provenientes da musculação, sendo desse número 7,69\% fazem outras atividades físicas além da musculação. De acordo com dados do estudo a grande parte das mulheres incontinentes, demonstraram impacto de moderado a muito grave na incontinência urinária. Outro estudo que obteve resultados significativos foi de Alves (2021), o qual teve 46 participantes, onde a perda de urina foi de constatada em $35,7 \%$, cerca de 15 mulheres e através do questionário do tipo International Consultation on Incontinence Questionnaire - Short Form (ICIQ-SF) que obteve como resultado que cerca de 5,53\% garantiram que tem impactos significativos na qualidade de vida. 
Além disso, estudo de Battistel (2020), relatou um grupo amostral de 220 mulheres sendo 15 mulheres tiveram IU com a prática da musculação, tendo assim aspectos relevantes no estudo, tendo a presença de IU entre as mulheres tabagistas que praticavam musculação foi de $6,2 \%$ e de $11,8 \%$ em mulheres que haviam sido submetidas a procedimentos ginecológicos, cauterização de lesão em colo uterino, ooforectomia unilateral, inserção de DIU e colposcopia. Também foi identificado que 75\% das mulheres que apresentavam IMC maior ou igual a $25 \mathrm{~kg} / \mathrm{m} 2$ apresentaram IU e que 53,3\% das mulheres que apresentaram perda urinária tinham idade inferior a 35 anos. Ou seja, seu estudo, apesar do grupo que praticou musculação apresentar 6,8\% de IU estavam diretamente ligadas ao IMC, procedimentos ginecológicos, tabagismo, inserção de DIU, e outros processos que tiverem relação na perna de urina no ato da execução do exercício.

Diferentemente dos estudos anteriores o levantamento de Amorim et al., 2019 o qual teve 30 praticantes de musculação, variando de 20 a 43 anos de idade, tendo como resultado de incidência de IU em dezesseis e a média do resultado do ICIQ-SF foi de 6,8 que caracteriza impacto moderado, e no que diz respeito ao tipo de IU, doze (75\%) praticantes de musculação apresentaram incontinência urinária de esforço, três $(18,8 \%)$ incontinência urinária de urgência, e, uma $(6,2 \%)$ incontinência urinária mista. Com isso, as mulheres submetidas ao estudo relataram o impacto causado pela IU sendo como impacto grave ou muito grave $50 \%$ das mulheres praticantes de musculação, ou seja, das incontinentes (8) não tem uma boa qualidade de vida.

Em contraponto ao estudo anterior onde foi realizado na cidade de Vitória da Consquista na Bahia o levantamento quantitativo sobre a IU nas academias de Belém do Pará com os autores Silva et al., 2018 constatou a prevalência de IU baixa na musculação, pois do grupo de 56 mulheres apenas 12 apresentaram perda de urina. Vale ressaltar que o estudo abordou voluntárias que praticavam musculação mais de 5 vezes na semana, incluindo finais de semana. No entanto, apesar do estudo ter números baixos de prevalência de IU, o esforço físico causado pela musculação tem sido apontado como um fator risco para adquirir algum tipo de disfunção urinária.

Ratificando os números, a pesquisa de Melo et al., 2018 constatou em 30 praticantes de musculação que 9 mulheres tinham IU decorrente da atividade física, tendo assim como resultados obtidos no presente estudo mostram que não houve associação, significância estatística entre incontinência urinaria em mulheres praticantes de musculação. Além disso, o estudo de Nunes et al., 2017 que teve 39 mulheres praticantes de musculação sendo que 7 constataram ter IU no ato da prática do exercício sendo que todas tem no dia a dia, constatando assim um número importante, o qual a IU constatada na musculação deve ter relação com quem já tem a IU, tendo assim o exercício físico como um agravante.

Ademais, Naves et al., 2016 que apresentaram um estudo composto por 42 mulheres, sendo que os sintomas de incontinência urinária de esforço foram encontrados em 12 participantes, sendo que dessas 58,33\% possuíam filhos, sendo assim $42,85 \%$ o tipo de parto foi o vaginal e em 57,15\% cesariana, fator segundo Naves et al., 2016 foi de grande importância do estudo da IU. Segundo Moreno (2008), o parto vaginal é a razão mais comum de disfunção do assoalho pélvico, além da média de partos por pessoa. Estudos mostram que a pressão e estiramento das delicadas estruturas pélvicas geradas pela tentativa de passagem do feto pelo canal vaginal e pela própria saída do mesmo são consideradas as principais razões de danos ao mecanismo de continência. Com isso, o estudo ao analisar 42 mulheres praticantes de atividade física, observou que a incidência de perda de urina foi presente em $28,57 \%$ das participantes, este número demonstra que a prática de atividades físicas pode ser um fator de risco importante para o desenvolvimento da Incontinência Urinária em mulheres, principalmente quando essas atividades são musculação e jump.

Por fim, estudo de Prigol et al., 2014 relataram que em 172 mulheres foram constatadas em 14 participantes a IU, ou seja, 8,1\% e no mesmo ano foram realizado o levantamento de Patrizzi et al., 2014 considerou 108 mulheres e cerca de 46 constataram a IU por conta de atividade física, grande parte praticantes de musculação, números esses maior que $40 \%$ das entrevistadas. Isso se deve aos elevados esforços, como é o caso dessa modalidade, podendo gerar um aumento da pressão intra-abdominal, sobrecarregando os órgãos da pelve e, consequentemente, ocorrer danos na musculatura encarregada por esses órgãos, causando 
assim a IU, além do mais, o excesso desse exercício pode provocar fadiga dos músculos do assoalho pélvico, predispondo assim a IU.

Sendo assim, a incontinência urinária de esforço (IUE) apresentada durante a atividade física é desenvolvida pela perturbação do mecanismo normal da continência ocasionada pelos aumentos excessivos na pressão intra-abdominal que acontecem durante o a realização do exercício. Com isso, nos estudos em questão foram reunidas 918 mulheres, sendo que 152 constataram a IU na musculação conforme Figura 1 abaixo.

Figura 1 - Amostras totais do estudo, praticantes de musculação com a IU detectada.

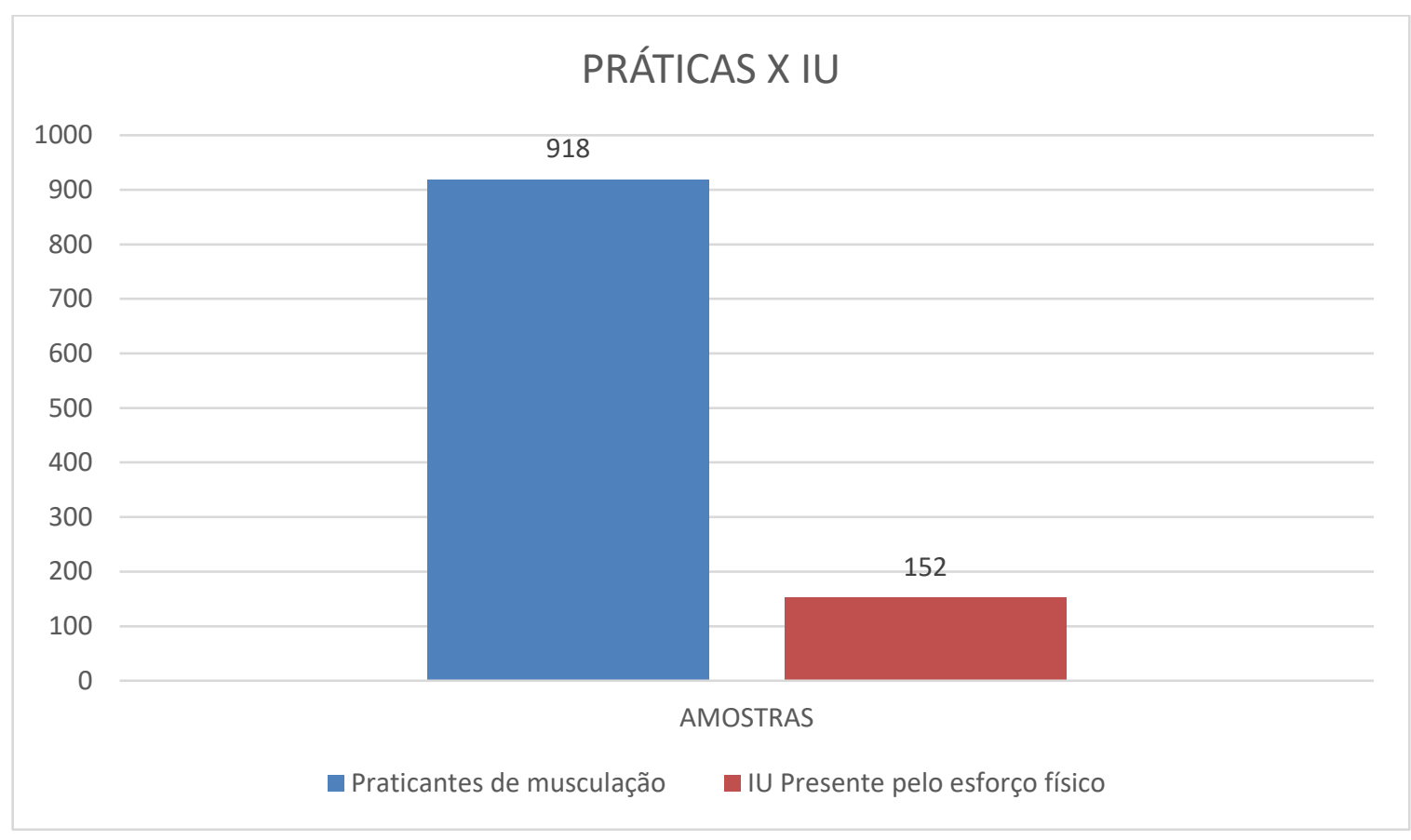

Fonte: Silva, Nascimento e Alencar (2021).

\section{Considerações Finais}

De modo geral, podemos observar que a pesquisa, apesar da limitação de amostra, os sinais e sintomas da incontinência urinária estavam presentes nas mulheres praticantes de musculação evidenciando o impacto de grave a muito grave ocasionado na qualidade de vida das mulheres participantes da pesquisa em questão. Contudo, é importante a realização de novos estudos com um maior grupo de mulheres, para assim ter uma melhor adequação de execução do exercício. Sendo assim, é necessário enfatizar que os profissionais que atuam nessa área devem elaborar melhores estratégias e formas mais adequadas no planejamento de treino de suas alunas. Com isso, recomenda-se realizar mais estudos detalhados com maiores números de mulheres, tendo assim um maior número de amostras, para assim ter resultados o suficiente para realizar estudos para mitigar e amenizar a prevalência de IU em mulheres praticantes de musculação.

\section{Agradecimentos}

Agradecemos à Deus primeiramente pela oportunidade proporcionada ao longo da caminhada acadêmica de conhecer essa área tão linda e maravilhosa. Segundo aos nossos familiares, por nos apoiar e compreender em todos os momentos e a nossa orientadora Indiara de Alencar, pelas orientações e ensinamentos. 


\section{Referências}

Alves, F. (2021). Sintomas de perda urinária em mulheres praticantes de exercícios físicos de alto e baixo impacto Pontifícia Universidade Católica de Goiás.

Amorim, L., Saraiva, D., \& Cirqueira, R. (2019). Prevalência de Incontinência Urinária em Mulheres Praticantes de Pilates e de Musculação. Revista Multidisciplinar e de Psicologia, 13(48), 1-12.

Battistel, G. (2020). Diferença na prevalência de incontinência urinária entre mulheres praticantes de musculação ou crossfit. Universidade do Sul de Santa Catarina

Borghetti, M., da Costa, M. V., \& Vasconcellos, N. (2011). Análise dos sinais e sintomas da incontinência urinária de esforço em mulheres de 25 a 50 anos praticantes de atividades físicas em academias. Ensaios e Ciência: Ciências Biológicas, Agrárias e da Saúde, 15(1), 1-14.

Cândido, F., Matnei, T., Galvão, L., Santos, V., Santos, M., Sarris, A., \& Sobreiro, B. (2017). Incontinência urinária em mulheres: breve revisão de fisiopatologia, avaliação e tratamento. 18(3), 2-14.

Ferraz, F. d., Ferraz, A., Nunes, E., \& Latorre, G. (2018). Prevalência de incontinência urinária em atletas praticantes de corrida de rua. 16(57), 37-44.

Guerra, T., Rossato, C., Nunes, E., \& Latorre, G. (2004). Atuação da fisioterapia no tratamento de incontinência urinaria de esforço. $42(6), 2-4$.

Henkes, D., Fiori, A., Carvalho, J., Tavares, K., \& Frare, J. (2015). Incontinência urinária: o impacto na vida de mulheres acometidas e o significado do tratamento fisioterapêutico. 36(2), 1-12.

Higa, R., Rivorêdo, C., Campos, L., Lopes, M., \& Turato, E. (2010). Vivências de mulheres brasileiras com incontinência urinária. 19(4), 627-635.

Lopes, M. H., \& Higa, R. (2006). Restrições causadas pela incontinência Restrições causadas pela incontinência urinária à vida da mulher urinária à vida da mulher. 40(1), 1-8.

Melo, A., \& Cirqueira, R. (2018). Incontinência Urinária em Mulheres Praticantes de Musculação. Revista Multidisciplinar e de Psicologia, $12(42), 1-11$.

Moreno, A. (2008). Fisioterapia em uroginecologia (2a ed.). Manole. (Obra original publicada em 2004)

Mourão, L., Luz, M., Marques, A., Benício, C., Nunes, B., \& Pereira, A. (2017). Caracterização e Fatores de Risco de Incontinência Urinária em Mulheres Atendidas em uma Clínica Ginecológica. 15(2), 82-91.

Naves, P., s Letieri, R., Simon, I., Leite, S., \& Letieri, M. (2016). Avaliação da qualidade de vida em mulheres com sintomas de incontinência urinária de esforço praticantes de atividade física. Movimento \& saúde, 8(1), 1-7.

Nunes, I., \& Brunazo, P. (2017). prevalência de incontinência urinária de esforço e função dos músculos do assoalho pélvico em mulheres praticantes de jump ou musculação.

Patrizzi, L., Viana, D., Silva, L., \& Pegorari, M. (2014). Incontinência urinária em mulheres jovens praticantes de exercício físico. $22(3)$, 1-6.

PrigoL, S., Sebben, V., \& Guedes, J. M. (2014). Prevalência da incontinência urinária em mulheres praticantes de atividade física nas academias da cidade de erechim. 38(141), 1-10.

Silva, L., Santos, W., Araujo, N., Rodrigues, C., \& Nunes, E. (2017). Disfunções urinárias em mulheres praticantes de atividade física em academias - um estudo transversal. 8(1), 1-8.

Silva, M., Porto, N., Santos, G., Sousa, J., Dantas, K., \& Pinheiro, V. (2019). Prevalência da incontinência urinária e seu impacto sobre a qualidade de vida de idosas atendidas em uma clínica escola de fisioterapia. 19(1), 203-2019.

Sousa, D., Dantas, A. C., Neves, N., Rodrigues, G., Santos, M., Tavares, A. M., Santos, A. D., Aquino, V., Melo, L., Silva, L., Santos, Y., Andrade, F., Martins, C., Aquino, M. J., Oliveira, D., \& Santana, L. (2021). Impacto dos sinais e sintomas de incontinência urinária na qualidade de vida de mulheres praticantes de musculação no Estado de Sergipe. 10(10), Artigo e598101018609. 